\title{
An exact solution for a rotating black hole in modified gravity
}

\author{
Francesco Filippini, Gianmassimo Tasinato
}

Department of Physics, Swansea University, Swansea, SA2 8PP, United Kingdom

\begin{abstract}
Exact solutions describing rotating black holes can offer important tests for alternative theories of gravity, motivated by the dark energy and dark matter problems. We present an analytic rotating black hole solution for a class of vector-tensor theories of modified gravity, valid for arbitrary values of the rotation parameter. The new configuration is characterised by parametrically large deviations from the Kerr-Newman geometry, controlled by non-minimal couplings between vectors and gravity. It has an oblate horizon in Boyer-Lindquist coordinates, and it can rotate more rapidly and have a larger ergosphere than black holes in General Relativity (GR) with the same asymptotic properties. We analytically investigate the features of the innermost stable circular orbits for massive objects on the equatorial plane, and show that stable orbits lie further away from the black hole horizon with respect to rotating black holes in GR. We also comment on possible applications of our findings for the extraction of rotational energy from the black hole.
\end{abstract}

\section{Introduction}

The new era of gravitational wave astronomy opens new opportunities for investigating with great precision the physics and dynamics of extreme compact objects, as black holes and neutron stars (see e.g. [1]). It will allow us to study for the first time the properties of fundamental interactions in a strong gravity regime, and test theories of gravity that are alternative to Einstein General Relativity (GR) [2]. The mysteries of cosmological dark energy and dark matter motivates attempts to modify GR. For example, it is important to explore the possibility to find theories admitting accelerating cosmological solutions with no need of a small positive cosmological constant (see e.g. [3]). The theoretically most interesting frameworks include scenarios automatically equipped with screening features, as chameleon [4] or Vainshtein [5] mechanisms. Screening mechanisms are able to locally hide the effects of additional light degrees of freedom besides GR's spin-2 field, and reproduce the predictions of Einstein gravity in a weak-field, spherically symmetric regime: see [6] for a review. The study of the properties of black hole solutions in these scenarios can provide new strong gravity tests for these theories, possibly manifesting sizeable deviations from GR. We focus here on theories with additional degrees of freedom non-minimally coupled with gravity through derivative interactions. Such interactions are essential for an implementation of Vainshtein screening mechanism. In the scalar-tensor case, the prototypes for such set-up are Galileons [7] and Horndeski [8] theories. The study of spherically symmetric black hole solutions in these scenarios have lead various interesting results, reviewed e.g. in [9]. We focus here on vector-tensor versions of these theories, dubbed vector Galileons, or generalized Proca [10-12]. Various examples of static, spherically symmetric black hole configurations have been found, and the study of compact objects as neutron stars have been recently developed [13,14].

In this work, we present and study examples of rotating black hole solutions with regular horizons for vector Galileons. Rotating black hole configurations which deviate from the Kerr family are hard to obtain in any theory of gravity, and only few examples of exact solutions are known in modified gravity frameworks. Solutions are known for scalar-tensor theories [15,16], also with a complex scalar [17], and in the context of 
Einstein dilaton Gauss-Bonnet theories [18,19]. Slowly rotating solutions in Horndeski theories are discussed in [20]. See [21] for a comprehensive review, and $[22,23]$ for useful parameterisations of possible deviations from the Kerr family of black holes in the context of arbitrary theories of gravity. Yet, given the fact that most astrophysical black holes are spinning, it is important to pursue the effort to determine and analyze explicit rotating black hole configurations in theories alternative to General Relativity. An additional theoretical reason to study rotating configurations is the fact that these objects break spherical symmetry (being at most axially symmetric). Hence they are an ideal set-up to start investigating screening mechanisms - as for example the Vainsthein mechanism - that are well studied and are known to be efficient only for spherically symmetric systems.

We determine exact solutions describing rotating black holes, by applying a disformal transformation on a version of the Kerr-Newman (KN) solution of the Einstein-Maxwell theory of gravity. The resulting configuration solves the equations of motion associated to a particular vector Galileon action, and is parameterically different from a KN system. Having exact solutions allows us to analytically investigate distinctive properties of spinning black holes in our theory. Our configurations are characterized by three asymptotic charges, the black hole mass, angular momentum, and vector charge. We show that the black hole horizon is oblate in Boyer-Lindquist coordinates, since its radial position depends on the polar angle. This is a feature that can make our black hole distinguishable from KN solutions, whose horizon lies at constant value of the radius in such coordinate system. The black hole maximal spin can be parametrically larger than KN configurations, for the same values of the asymptotic charges. The solution admits also a 'massless' limit of black hole with zero mass, but with a vector charge which ensures the existence of a regular horizon. The study of equatorial circular trajectories admits an analytical treatment. We show that probe massive objects can rotate faster than in the KN family of solutions. Innermost stable circular orbits (ISCOs) lie further away from the black hole horizon with respect to rotating black holes in GR. We also comment on possible applications of our findings for the extraction of rotational energy from the black hole.

\section{Set-up}

We build a modification of Einstein gravity which includes additional vector degrees of freedom, belonging to the class of theories dubbed vector-tensor Galileons [10-12]. Such vector degrees of freedom can be associated with dark forces motivated by dark matter or dark energy model building. Our aim will be to investigate new rotating black hole solutions for the theory we consider.

In order to construct a modified gravity action, our starting point is a standard Einstein-Maxwell system, described by an action

$$
S_{E M}=\int d^{4} x \sqrt{-\tilde{g}}\left[\frac{\tilde{R}}{4}-\frac{1}{4} \tilde{F}^{\mu \nu} \tilde{F}_{\mu \nu}\right] .
$$

Although we call the previous action an 'Einstein-Maxwell' system, as we are going to discuss the vector fields appearing in eq (1) should not be identified with standard electromagnetism, but with additional dark vector forces. We use a mostly plus metric signature, and Weinberg's conventions for the Riemann and derived tensors. Our results are expressed in natural units, setting $c=1, \hbar=1$, and $4 \pi G=1$. The theory enjoys an Abelian gauge invariance, $A_{\mu} \rightarrow A_{\mu}+\partial_{\mu} \xi$ for any arbitrary function $\xi$. It propagates four degrees of freedom, two in the transverse traceless tensor sector, and two in the transverse vector sector. Exact black hole solutions for the equations of motion associated for this theory are well studied, and include static Reissner-Nordström and rotating Kerr-Newman (KN) configurations. They satisfy no-hair theorems, which state that black holes are uniquely defined in terms of their mass, angular momentum, and charge.

To generate new solutions, we act on this action with a disformal transformation [24-26] involving vector fields and parameterized by a real constant $\beta$, which plays a key role in what follows:

$$
\tilde{g}_{\mu \nu}(x)=g_{\mu \nu}(x)-\beta^{2} A_{\mu}(x) A_{\nu}(x),
$$




$$
\tilde{A}_{\mu}(x)=A_{\mu}(x)+\partial_{\mu} \alpha(x),
$$

for any arbitrary function $\alpha(x)$ (the transformation of the vector simply reflects the gauge freedom of the original theory, and $\alpha$ does not appear in the final formulae). More general disformal/conformal transformations can be considered (see for example [26]), but transformation (2) is sufficient for our purposes. The disformed metric is invertible, with inverse given by

$$
\tilde{g}^{\mu \nu}=g^{\mu \nu}+\beta^{2} \gamma_{0}^{2} A^{\mu} A^{\nu} \quad \text { with } \quad \gamma_{0}^{2}=\frac{1}{1-\beta^{2} A^{\mu} A_{\mu}} .
$$

Up to total derivatives, the disformed action reads

$$
S_{\text {disf }}=S_{E H}+S_{\text {matt }}=\int d^{4} x \sqrt{-g}\left(\mathcal{L}_{E H}+\mathcal{L}_{\text {matt }}\right),
$$

with Lagrangian densities

$$
\mathcal{L}_{E H}=\frac{1}{4 \gamma_{0}}\left[R-\frac{\beta^{2}}{4} \gamma_{0}^{2}\left(S_{\mu \nu} S^{\mu \nu}-S^{2}\right)+\frac{\beta^{2}}{4} F_{\mu \nu} F^{\mu \nu}+\frac{\beta^{4}}{2} \gamma_{0}^{2} F_{\mu \rho} F_{\nu}^{\rho} A^{\mu} A^{\nu}\right]
$$

and

$$
\mathcal{L}_{\text {matt }}=-\frac{1}{4 \gamma_{0}}\left[F^{\mu \nu} F_{\mu \nu}+2 \beta^{2} \gamma_{0}^{2} F_{\mu \rho} F_{\nu}^{\rho} A^{\mu} A^{\nu}\right]
$$

with

$$
\begin{aligned}
& F_{\mu \nu}=\nabla_{\mu} A_{\nu}-\nabla_{\nu} A_{\mu}, \\
& S_{\mu \nu}=\nabla_{\mu} A_{\nu}+\nabla_{\nu} A_{\mu}, \quad S=S_{\mu \nu} g^{\mu \nu} .
\end{aligned}
$$

In a more compact notation:

$$
S_{\text {disf }}=\int d^{4} x \sqrt{-g} \frac{1}{4 \gamma_{0}}\left[R-\frac{\beta^{2}}{4} \gamma_{0}^{2}\left(S_{\mu \nu} S^{\mu \nu}-S^{2}\right)-\frac{4-\beta^{2}}{4} F_{\mu \nu} F^{\mu \nu}+\frac{\beta^{4}-4 \beta^{2}}{2} \gamma_{0}^{2} F_{\mu \rho} F_{\nu}^{\rho} A^{\mu} A^{\nu}\right] .
$$

The system after the disformal transformation describes a vector-tensor theory of gravity which deviates from the usual Einstein-Maxwell case of eq (1) by quantities depending on the disformal parameter $\beta^{2}$. Action (9) will be our modified gravity theory of reference in this work. It contains non-minimal couplings of the vector to gravity, and derivative self-interactions of the form that usually characterize Horndeski systems. The theory is free of Ostrogradsky instabilities. In fact, this system belongs to the class of theories dubbed vector-tensor Galileons or generalized Proca, which have been investigated at length for their distinctive properties for cosmology [27], field theory [28], and black holes [13,14]. Disformal transformations are known to preserve the causality properties of the theory one starts with [24]: hence since the Einstein-Maxwell theory is causally well behaved, one does not expect instabilities or causal pathologies in the theory described by eq (9).

Although the two actions (1) and (9) are related by a disformal transformation, they are not equivalent when additional matter, minimally coupled with gravity, is included into the system. Hence, interpreting the system of (9) as part of a more general action including matter fields, we can expect that its physical consequences can be different from an Einstein-Maxwell set-up. We will elaborate on these points in the next Sections.

Action (9) breaks an Abelian gauge invariance, since the Lagrangian depends explicitly on the gauge potential. On the other hand, it inherits some memory of the original gauge symmetry of action (1). In fact, the final action is invariant, up to total derivatives, under the more general gauge transformation

$$
\begin{aligned}
g_{\mu \nu} & \rightarrow g_{\mu \nu}+\beta^{2} \partial_{\mu} \chi A_{\nu}+\beta^{2} \partial_{\nu} \chi A_{\mu}+\beta^{2} \partial_{\mu} \chi \partial_{\nu} \chi, \\
A_{\mu} & \rightarrow A_{\mu}+\partial_{\mu} \chi,
\end{aligned}
$$


with $\chi$ an arbitrary scalar function. It is easy to check that the quantities $\tilde{g}_{\mu \nu}$ and $\tilde{A}_{\mu}$, eqs (2) and (3), are invariant under the simultaneous transformations (10), (11). Hence the final action, which can be expressed in terms of the combinations $\tilde{g}_{\mu \nu}$ and $\tilde{A}_{\mu}$, is invariant under this symmetry. Notice that the limit $\beta \rightarrow 0$ reduces, as expected, to standard Abelian gauge symmetry. Such symmetry ensures that the final action propagates four dynamical modes. The absence of a fifth, scalar degree of freedom is a welcoming feature for phenomenology, since it can automatically avoid stringent constraints on the existence of long range scalar fifth forces. In this work, we focus on determining and analysing new regular rotating black holes for the theory (9), equipped with the new gauge symmetry (10), (11), obtained by a disformal transformation of the Einstein-Maxwell action.

\section{New rotating solutions in vector-tensor theories of gravity}

In this Section we show that a disformal transformation acting on an appropriate solution of the original field equations leads to a new regular, rotating black hole for action (9), with a non-trivial profile for the vector field turned on. One might expect that the non-linearity of field equations, and the fact that we renounce to spherical symmetry, imply that rotating configurations in this theory are different from GR solutions. We confirm this expectation, showing that rotating configurations with non-trivial vector profiles have specific properties that make them distinguishable from their GR counterparts. This fact can lead to qualitatively new ways to test modified gravity models, by investigating the properties of their black hole solutions.

We start from a Kerr-Newman solution of the Einstein-Maxwell action (1), which can be expressed in Boyer-Lindquist coordinates as

$$
\begin{aligned}
& d s^{2}=\left(\frac{d r^{2}}{\Delta}+d \theta^{2}\right) \rho^{2}-\left(d t-a \sin ^{2} \theta d \phi\right)^{2} \frac{\Delta}{\rho^{2}}+\left[\left(r^{2}+a^{2}\right) d \phi-a d t\right]^{2} \frac{\sin ^{2} \theta}{\rho^{2}} \\
& A_{\mu}=\left\{-\frac{Q r}{\rho^{2}}, A_{r}(r), 0, \frac{a Q r \sin ^{2} \theta}{\rho^{2}}\right\},
\end{aligned}
$$

with:

$$
\begin{aligned}
& \Delta=a^{2}+r^{2}-2 M r+Q^{2}, \\
& \rho^{2}=r^{2}+a^{2} \cos ^{2} \theta .
\end{aligned}
$$

This configuration describes a rotating charged black hole. See e.g. [29, 30] for comprehensive reviews on rotating black hole solutions in GR. The constants $M, Q$, and $a$ are associated with the black hole mass, charge, and angular momentum. We turn on an arbitrary radial component for the vector potential, which we denote with $A_{r}(r)$ : since the original Einstein-Maxwell action respects an Abelian gauge symmetry, $A_{\mu} \rightarrow A_{\mu}+\partial_{\mu} \chi$, the radial profile of $A_{r}(r)$ does not affect the geometry, and can in principle be 'gauged away'. On the other hand, it plays an important role for our purposes, since the disformed transformation mixes metric and vector degrees of freedom, and does not enjoy the standard Abelian symmetry.

We apply the disformal transformation of eq (2) to eqs (12), (13). The resulting configuration is solution of the vector-tensor theory (9), describing a rotating system. Potential problems arise though, since the resulting geometry generically has naked singularities or other pathologies not covered by horizons. Alternatively it is not asymptotically flat, or it results too complicated to be of any use. On the other hand, the radial vector profile $A_{r}(r)$ in eq (13) influences the geometry after the disformal transformation, and we can use this fact at our advantage. We determined a specific profile for the radial vector component, which leads to a regular, asymptotically flat black hole configuration:

$$
A_{r}(r)=\frac{Q r}{\Delta(r)}
$$


It would be interesting to understand whether other radial vector profiles give regular solutions. With this choice for the radial vector component, the disformed metric contains off-diagonal components $d r d t$ and $d r d \phi$ components and the final metric reads

$$
d s^{2}=g_{t t} d t^{2}+g_{r r} d r^{2}+g_{\theta \theta} d \theta^{2}+g_{\phi \phi} d \phi^{2}+2 g_{t r} d t d r+2 g_{t \phi} d t d \phi+2 g_{r \phi} d r d \phi,
$$

with

$$
\begin{aligned}
g_{t t} & =-1-\frac{\left(Q^{2}-2 M r\right)\left(r^{2}+a^{2} \cos ^{2} \theta\right)-\beta^{2} Q^{2} r^{2}}{\left(r^{2}+a^{2} \cos ^{2} \theta\right)^{2}} \\
g_{t r} & =\frac{-\beta^{2} Q^{2} r^{2}}{\left(a^{2}+r^{2}-2 M r+Q^{2}\right)\left(r^{2}+a^{2} \cos ^{2} \theta\right)} \\
g_{t \phi} & =a \sin ^{2} \theta \frac{\left(Q^{2}-2 M r\right)\left(r^{2}+a^{2} \cos ^{2} \theta\right)-\beta^{2} Q^{2} r^{2}}{\left(r^{2}+a^{2} \cos ^{2} \theta\right)^{2}} \\
g_{r r} & =\frac{\left(a^{2}+r^{2}-2 M r+Q^{2}\right)\left(r^{2}+a^{2} \cos \theta^{2}\right)+\beta^{2} Q^{2} r^{2}}{\left(a^{2}+r^{2}-2 M r+Q^{2}\right)^{2}} \\
g_{r \phi} & =\frac{\beta^{2} a Q^{2} r^{2} \sin ^{2} \theta}{\left(a^{2}+r^{2}-2 M r+Q^{2}\right)\left(r^{2}+a^{2} \cos ^{2} \theta\right)} \\
g_{\theta \theta} & =r^{2}+a^{2} \cos ^{2} \theta \\
g_{\phi \phi} & =\sin ^{2} \theta\left[r^{2}+a^{2}-a^{2} \sin ^{2} \theta \frac{\left(Q^{2}-2 M r\right)\left(r^{2}+a^{2} \cos ^{2} \theta\right)-\beta^{2} Q^{2} r^{2}}{\left(r^{2} \theta\right)^{2}}\right] .
\end{aligned}
$$

In a more compact notation we can write

$$
\begin{aligned}
d s^{2}= & -1-\frac{\left(Q^{2}-2 M r\right) \rho^{2}-\beta^{2} Q^{2} r^{2}}{\rho^{4}} d t^{2}-\frac{\beta^{2} Q^{2} r^{2}}{\Delta \rho^{2}} d t d r+a \sin ^{2} \theta \frac{\left(Q^{2}-2 M r\right) \rho^{2}-\beta^{2} Q^{2} r^{2}}{\rho^{4}} d t d \phi+\frac{\Delta \rho+\beta^{2} Q^{2} r^{2}}{\Delta^{2}} d r^{2} \\
& +\frac{\beta^{2} a \sin ^{2} \theta Q^{2} r^{2}}{\Delta \rho^{2}} d r d \phi+\rho^{2} d \theta^{2}+\sin ^{2} \theta\left[r^{2}+a^{2}-a^{2} \sin ^{2} \theta \frac{\left(Q^{2}-2 M r\right) \rho^{2}-\beta^{2} Q^{2} r^{2}}{\rho^{4}}\right] d \phi^{2}
\end{aligned}
$$

with $\Delta, \rho$ given in eqs (14), while the gauge field components read

$$
\begin{aligned}
A_{\mu} & =\left(A_{t}, A_{r}, A_{\theta}, A_{\phi}\right) \\
& =\left(-\frac{Q r}{\rho^{2}}, \frac{Q r}{\Delta}, 0, \frac{Q r a \sin ^{2} \theta}{\rho^{2}}\right) .
\end{aligned}
$$

This geometry describes a rotating black hole with regular horizon, charged under the vector field:

- the dimensionless quantity $\beta^{2}$ parametrically controls deviations from the Kerr-Newman geometry in eq (18). The geometry depends on three integration constants, $M, a$, and $Q$, as well as by the parameter $\beta^{2}$ associated the non-minimal couplings of vector to gravity in action (9). Besides mass and spin, the geometry is charged under the vector degrees of freedom that control our modification of gravity (and should not be identified with electromagnetism). As we shall learn in the next Sections, the contributions depending on $\beta$ modify the structure of the black hole horizons, and has consequences for the properties of geodesics of massive particles;

- the vector field profile (19) has three physical components turned on, against the two of the Kerr-Newman configuration. The vector radial component is physical in this case, and can not be gauged away without simultaneously changing the geometrical properties of the system.

Using the Mathematica package xAct [31], we explicitly checked that eqs (18) (19) are a solution of all the equations of motion associated with the disformed vector-tensor action. It is important to emphasise that although this configuration is disformally related to Kerr-Newman, it is a new solution for the vector-tensor modified gravity theory we are considering. The systems (1) and (9) can have distinct physical implications when additional matter is included, minimally coupled with gravity. For example, we will show in Section 5 that the features of time-like 
geodesics in this black hole background are different with respect to rotating black holes in GR. The expression for the Ricci scalar is

$$
R=2 \beta^{2} a^{2} Q^{2} \cos ^{2} \theta \frac{\left(a^{2} \cos ^{2} \theta-3 r^{2}\right)}{\left(r^{2}+a^{2} \cos ^{2} \theta\right)^{4}} .
$$

Since the Ricci scalar is non-vanishing, this configuration is different from the original KN solution, where this quantity is equal to zero. In Appendix A we show that asymptotic mass, charge and angular momentum for this configuration are $M, Q$, and $a / M$. The unique geometrical singularity associated with our new disformed solution (18) is the Kerr singularity at the locus $r^{2}+a^{2} \cos ^{2} \theta=0$. We also checked that, besides this singularity, the remaining curvature invariants, obtained contracting Ricci and Riemann tensors, are everywhere regular. The solution is asymptotically flat since the metric components approach flat space at asymptotic infinity, and the curvature invariants asymptotically tend to zero. In principle we can still use the gauge freedom of eq (10), (11) to change the profile of vector components, for example turning off the radial quantity $A_{r}$. However, this gauge operation changes the metric as well, and can lead to singular geometries. For this reason we work with metric and gauge field as expressed in eqs (18), (19). Before discussing in some detail the non-linear features of this disformed configuration, it is interesting to analyse the limit of small rotation: at first order in an expansion on the rotation parameter $a$, the geometry reads

$$
\begin{aligned}
d s^{2}= & \left(-1+\frac{2 M}{r}-\frac{\left(1-\beta^{2}\right) Q^{2}}{r^{2}}\right) d t^{2}+\frac{r^{4}}{\Delta^{2}}\left(1-\frac{2 M}{r}+\frac{Q^{2}\left(1+\beta^{2}\right)}{r^{2}}\right) d r^{2}+r^{2} d \theta^{2}+r^{2} \sin ^{2} \theta d \phi^{2} \\
& -\frac{2 \beta^{2} Q^{2}}{\Delta} d t d r+2 a \sin ^{2} \theta\left[-\frac{2 M}{r}+\frac{Q^{2}\left(1-\beta^{2}\right)}{r^{2}}\right] d t d \phi+\frac{2 \beta^{2} a Q^{2} \sin ^{2}(\theta)}{\Delta} d r d \phi,
\end{aligned}
$$

where $\Delta$ is calculated with $a^{2}=0$.

In the limit $a \rightarrow 0$, the metric becomes

$$
d s^{2}=\left(-1+\frac{2 M}{r}-\frac{\left(1-\beta^{2}\right) Q^{2}}{r^{2}}\right) d t^{2}+\frac{r^{4}}{\Delta^{2}}\left(1-\frac{2 M}{r}+\frac{Q^{2}\left(1+\beta^{2}\right)}{r^{2}}\right) d r^{2}+r^{2} d \theta^{2}+r^{2} \sin ^{2} \theta d \phi^{2}-\frac{2 \beta^{2} Q^{2}}{\Delta} d t d r
$$

which can be considered the disformal Reissner-Nordstrom solution.

It would be interesting to investigate whether the regime $\beta^{2}>1$ leads to problems for the system (e.g. instabilities of vector degrees of freedom around our geometry). But we do not touch these topics in this work, and explore the properties of the geometry for any value of $|\beta|$. In the next section we will perform an analytic study of the structure of horizons, which have features which make them distinguishable from their GR counterparts.

\section{Structure and properties of horizons}

We analyse the structure of the horizons, where departures from the Kerr geometry are manifest. The vector Lagrangian (9) is non-minimally coupled with gravity, and the non-linear derivative interactions induce qualitative deviations from GR rotating solutions. For example, we will learn that our black holes can rotate faster than their GR counterparts, for the same mass and charge.

Given a spherical coordinate system $\{t, r, \theta, \phi\}$, the event horizon corresponds to points where the hypersurfaces of constant $r$ become null, namely

$$
g^{\mu \nu} \partial_{\mu} r \partial_{\nu} r=g^{r r}=0,
$$

where $\partial_{\mu} r$ is the 1-form normal to constant $r$ hypersurfaces. A Killing horizon, instead, is the null hypersurface where the length of a Killing vector vanishes, $k^{\mu} k_{\mu}=0$. For stationary geometries as our configuration, the ergosphere corresponds to the Killing horizon of the time translation Killing vector $k^{\mu}=\{1,0,0,0\}$. A priori, ergosphere and event horizon are distinct hypersurfaces. See [32] for details. For the standard KN black hole (obtained from solution (18) setting $\beta^{2}=0$ ), the exterior event horizon and the ergosphere are situated at

$$
\begin{array}{r}
r_{h o r}^{K N}=M+\sqrt{M^{2}-a^{2}-Q^{2}}, \\
r_{\text {erg }}^{K N}=M+\sqrt{M^{2}-a^{2} \cos ^{2} \theta-Q^{2}} .
\end{array}
$$


Using Boyer-Lindquist coordinates, the event horizon is spherical in the Kerr geometry, being located to a radial position independent on the angular coordinates. (Although the intrinsic horizon geometry is actually squashed, as manifest using appropriate coordinate systems [32]). The ergosphere is instead an ellipsoid.

In the vector-tensor theory of gravity we are examining, the event horizons and the ergospheres are hypersurfaces of revolution around the azymuthal coordinate. Their positions is given by the real positive solutions of equations

$$
\begin{aligned}
& \text { Horizon: } \quad g^{r r}=0 \Rightarrow f_{h} \equiv\left(r^{2}+a^{2} \cos ^{2} \theta\right)\left[r^{2}-2 M r+a^{2}+Q^{2}\right]-\beta^{2} Q^{2} r^{2}=0, \\
& \text { Ergosphere: } \quad k^{\mu} k_{\mu}=0 \Rightarrow f_{e} \equiv\left(r^{2}+a^{2} \cos ^{2} \theta\right)\left[r^{2}-2 M r+a^{2} \cos ^{2} \theta+Q^{2}\right]-\beta^{2} Q^{2} r^{2}=0 .
\end{aligned}
$$

Equations $f_{h, e}=0$ are algebraic equations of fourth order in the coordinate $r$ : they can be solved analytically, but their solutions are complicated. Depending on whether the sign of the discriminant, they can have four, two or no real roots. We denote their maximal real roots, with $r_{h}$ and $r_{e}$ respectively: $r_{h}$ corresponds to the position of the external horizon of the black hole. Such expressions depend on the polar angle $\theta$ : in our configuration, neither the ergosphere nor the horizons have spherical shape in Boyer-Lindquist coordinates. This is a major qualitative difference with respect to Kerr-Newman black holes, which have spherical horizons in Boyer-Lindquist coordinates. We find that $r_{e} \geq r_{h}$ : substituting the value $r_{e}$ in the expression for $f_{h}$ (25), we find $f_{h} \geq 0$, hence the position of the ergosphere is outside the horizon. The horizon and ergosphere hypersurfaces touch at the poles, $\theta=0, \pi$, since at these points $f_{h, e}$ coincide. The only value of the polar angle where an expression for $r_{h, e}$ can be analytically obtained is the equator, where we get

$$
\begin{aligned}
& r_{h}(\theta=\pi / 2)=M+\sqrt{M^{2}-a^{2}-Q^{2}\left(1-\beta^{2}\right)}, \\
& r_{e}(\theta=\pi / 2)=M+\sqrt{M^{2}-Q^{2}\left(1-\beta^{2}\right)} .
\end{aligned}
$$

It is simple to show that the external horizon of Kerr-Newman black hole is always in the interior of the horizon of our disformal black hole configuration, for the same values of mass, charge and angular momentum. Substituting the value $r_{h}^{K N}$ for the position of the Kerr-Newman horizon in the expression (25) for $f_{h}$, we find that this quantity is negative, hence it lies inside the disformal black hole horizon. Fig. 1 represents the shape of black hole horizons and ergosphere for our systems, using Boyer-Lindquist coordinates.

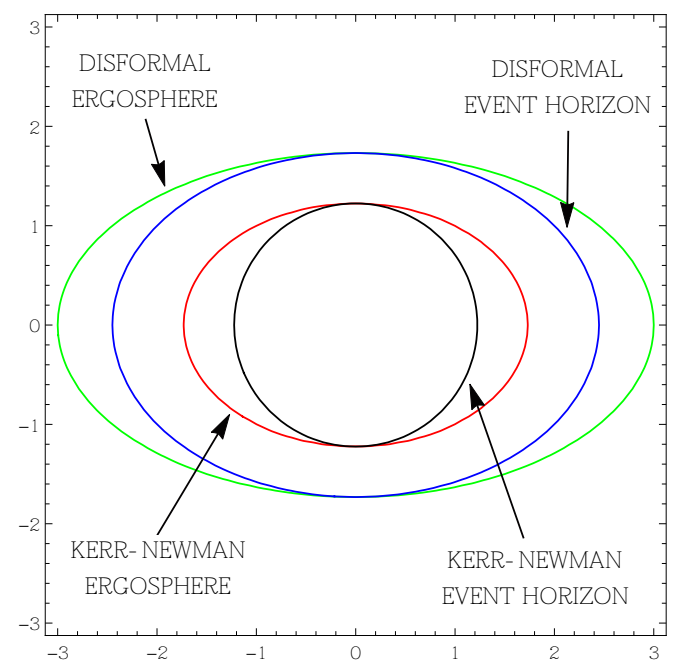

Figure 1: Pictorial representation of the shape of horizon and ergosphere for the disformed black hole using Boyer-Lindquist coordinates, as discussed in the main text. 
At this point it is interesting looking at the event horizon's angular velocity.

Let's consider a photon emitted along the $\phi$ direction at radial distance $r$; so, there are no components of the velocity along the $\theta$ and $r$ directions, and the metric simply reads

$$
d s^{2}=g_{t t} d t^{2}+2 g_{t \phi} d t d \phi+g_{\phi \phi} d \phi^{2}=0,
$$

which can be solved to obtain

$$
\frac{d \phi}{d t}=-\frac{g_{t \phi}}{g_{\phi \phi}} \pm \sqrt{\left(\frac{g_{t \phi}}{g_{\phi \phi}}\right)^{2}-\frac{g_{t t}}{g_{\phi \phi}}} .
$$

Evaluating this expression on the event horizon

$$
f_{h}=0
$$

the term in the square root term vanishes and it simply remains

$$
\left.\frac{d \phi}{d t}\right|_{r_{h}}=\Omega_{h}=\frac{a}{a^{2}+r_{h}^{2}}
$$

which is the angular velocity of the event horizon. Notice that since the position of the horizon depends on the angular coordinate $\theta$, being it solution of eq (25), the angular velocity is not constant on all the horizon surface.

\subsection{Maximal black hole spin and horizon oblateness}

The modifications of the geometry proportional to the parameter $\beta$ can deform the horizon, making it oblate also in Boyer-Lindquist coordinates. Interestingly, they can also allow for ultra spinning black holes, i.e. black hole configurations that rotate faster than Kerr. It is not easy to analytically study these properties of the geometry, a part from two special cases that we are going to investigate in this subsection.

\section{Case 1: The limit of large value of $\beta^{2}$}

The size of the disformal horizon increases with the polar angular coordinate: its smallest value is at the poles $\theta=0, \pi$, while its maximal value is at the equator $\theta=\pi / 2$. The existence of a regular horizon depends on the value of the black hole spin parameter $a$ : beyond an extremal value $a_{\max }$, an horizon ceases to exist. Such value of $a_{\max }$ can be found by studying the equation which gives the radial position of the horizon at the poles in Boyer-Lindquist coordinates $(\theta=0, \pi)$. We focus on the poles because at this position we get the most stringent condition on $a_{m a x}$. The radial horizon equation at the poles can be expressed as

$$
r^{4}-2 M r^{3}+\left(2 a^{2}+q M^{2}\right) r^{2}-2 a^{2} M r+a^{4}+\frac{a^{2} M^{2} q}{1-\beta^{2}}=0,
$$

where we define the combination

$$
q \equiv \frac{Q^{2}}{M^{2}}\left(1-\beta^{2}\right)
$$

which can be positive or negative, depending on the size of $\beta$. Analytic solutions for equation (32) are cumbersome: but a great simplification occurs in the limit $|\beta| \gg 1$. We consider this regime maintaining a fixed value for the parameter $q$ defined in eq (33). This implies that we simultaneously take a limit in which black hole charge $Q$ becomes smaller and smaller, so to maintain $q$ finite. This limit physically correspond to a regime of 'strong nonminimal coupling' between vector and gravity in the action (9).

In this regime, the last term in eq (32) can be neglected, and the equation admits a simple expression for its real roots: the external horizon sits at the position

$$
r_{h}^{p o l}=\frac{M}{2}\left(1+\sqrt{1-q}+\sqrt{2-q+2 \sqrt{1-q}-4 \frac{a^{2}}{M^{2}}}\right) .
$$

Requiring a positive argument for the last square root in the previous expression imposes an upper bound on the rotation parameter $a$ : its largest allowed value is

$$
a_{\max }=\frac{M}{2} \sqrt{2-q+2 \sqrt{1-q}}
$$




$$
=\frac{M}{2} \sqrt{2+\frac{Q^{2}}{M^{2}}\left(\beta^{2}-1\right)+2 \sqrt{1+\frac{Q^{2}}{M^{2}}\left(\beta^{2}-1\right)}},
$$

where in the second line we use the definition (33). When $\beta^{2}>1$, then $a_{\max } \geq M$ (with equality saturated for $Q=0$ ). Recall that $M$ is the maximal value of the spin $a$ for a Kerr black hole (while for Kerr-Newman the maximal spin is $\left.\sqrt{M^{2}-Q^{2}}<M\right)$. Hence when $\beta^{2}$ is large, our disformed rotating black hole can have angular momentum parametrically larger than in Einstein gravity. (Ultraspinning black holes are however possible in theories with more than $3+1$ dimensions [33] or in modified gravity theories including a complex scalar [17] or EdGB [19].)

We obtained this result in the extremal limit of very large $|\beta|$ : it is possible to check numerically that for smaller values of $|\beta|$, the value of $a_{\max }$ reduces its size with respect to (36), by a quantity that is proportional to $1 /|\beta|$. In any case, we will use $a_{\max }$ of eq (36) as reference for our discussion. We also checked that the conditions on the parameter $a$ for having a regular horizon are most stringent at the poles, and less restrictive at other angular positions. In other words, the horizon at the equator could in principle rotate faster than $a_{\max }$ of eq (35), but the requirement of having a regular horizon everywhere does not allow for this.

When the spin parameter attains the value $a_{\max }$, the ratio between the radial size of the horizon at the poles versus the size of the horizon at the equator quantifies the oblateness $\omega$ of the black hole in Boyer-Lindquist coordinates

$$
\omega=1-\frac{r_{h}^{p o l}}{r_{h}^{e q}}=1-\frac{1+\sqrt{1-q}}{2+\sqrt{2-3 q-2 \sqrt{1-q}}} .
$$

In the limit of large, negative values of $q$, oblateness approaches the extremal value $\omega_{\max }=1-1 / \sqrt{3}$ : hence in this limit the radial size of the horizon at the poles is $1 / \sqrt{3} \simeq 0.57$ times smaller than the size of the horizon at the equator, as measured in Boyer-Lindquist coordinates.

We can interpret the horizon properties we determined as due to our non minimal vector-tensor interactions, which are able to contrast strong centrifugal forces, and maintain a regular horizon even for large spins, at the price of deforming the horizon shape.

\section{Case 2: Massless black holes}

Interestingly, if $\beta^{2}>1$, our black hole configuration (18) admits a regular horizon even in the massless limit $M \rightarrow 0$. In this limit, the solution has two horizons: the radial position of the external one depends on the polar angle and is given by the expression (valid if $\beta^{2}>1$ )

$$
r_{h}^{2}=\frac{1}{2}\left[Q^{2}\left(\beta^{2}-1\right)-a^{2}\left(1+\cos ^{2} \theta\right)+\sqrt{\left[Q^{2}\left(\beta^{2}-1\right)-a^{2}\left(1+\cos ^{2} \theta\right)\right]^{2}-4 a^{2}\left(a^{2}+Q^{2}\right) \cos ^{2} \theta}\right] .
$$

The radial size of the black hole is maximal at the equator $(\theta=\pi / 2)$ and minimal at the poles $(\theta=0, \pi)$. The condition of having a regular horizon at the pole imposes an upper bound on the black hole spin parameter, given by

$$
a_{\max }^{2}=Q^{2} \frac{\left(\beta^{2}-1\right)^{2}}{4 \beta^{2}} .
$$

It is also simple to compute the black hole oblateness for extremal values of the black hole spin, as done in Case 1 around eq (37). In this case we obtain

$$
\omega=1-\sqrt{\frac{1+\beta^{2}}{1+3 \beta^{2}}} .
$$

Also here the maximal oblateness is $1-1 / \sqrt{3}$, showing that for large $\beta$ the radial size of the horizon at the poles is $1 / \sqrt{3} \simeq 0.57$ times smaller than the size of the horizon at the equator.

\section{Equatorial orbits}

The dynamics of massive and massless fields orbiting around rotating black holes is a broad subject with several ramifications and applications to astrophysics and cosmology, and it is the first step towards the study of black hole 
accretion disks, or of the extraction of rotational energy from spinning black holes. See [29] for an enlightening review, and [34] for the original paper studying this family of orbits in Kerr configurations. We focus our attention to circular orbits for massive particles in the equatorial plane, examining features that are peculiar of our disformed rotating black hole. We make the hypothesis that the particles are only minimally coupled with gravity. Since the geometry is axially symmetric, stable orbits exist which remain confined on the equatorial plane. Having an exact form for the metric allows us to point out distinctive properties of equatorial orbits by simple, analytical considerations, which are a natural generalization of arguments developed for the Kerr(/Newman) geometry [34].

The disformal metric (18) on the equatorial plane $(\theta=\pi / 2)$ reads

$$
\begin{aligned}
d s^{2}=g_{t t} d t^{2}+g_{r r} d r^{2}+ & g_{\theta \theta} d \theta^{2}+g_{\phi \phi} d \phi^{2}+2 g_{t r} d t d r+2 g_{t \phi} d t d \phi+2 g_{r \phi} d r d \phi . \\
g_{t t} & =-\frac{r(r-2 M)+\left(1-\beta^{2}\right) Q^{2}}{r^{2}} \\
g_{r r} & =\frac{r^{2}\left[a^{2}+r(r-2 M)+\left(1+\beta^{2}\right) Q^{2}\right]}{\Delta^{2}} \\
g_{\theta \theta} & =r^{2} \\
g_{\phi \phi} & =r^{2}+\frac{a^{2}\left[r(2 M+r)-\left(1-\beta^{2}\right) Q^{2}\right]}{r^{2}} \\
g_{t r} & =-\frac{\beta^{2} Q^{2}}{\Delta} \\
g_{t \phi} & =\frac{a\left[\left(1-\beta^{2}\right) Q^{2}-2 M r\right]}{r^{2}} \\
g_{r \phi} & =\frac{\beta^{2} a Q^{2}}{\Delta}
\end{aligned}
$$

Such metric is independent from $t$ and $\phi$, hence we can define the conserved energy per unit mass e and the conserved angular momentum per unit mass $\ell$ along the symmetry axis:

$$
\begin{aligned}
& e \equiv-k^{\mu} u^{\nu} g_{\mu \nu}, \\
& \ell \equiv r^{\mu} u^{\nu} g_{\mu \nu},
\end{aligned}
$$

where $u^{\mu}$ is the 4 -velocity vector, and $k^{\mu}$ and $r^{\mu}$ are the Killing vectors defined in Appendix A. Using the metric (41), the previous equations can be expressed in the following way:

$$
\begin{aligned}
& e=-\left(g_{t t} u^{t}+g_{t \phi} u^{\phi}\right), \\
& \ell=g_{\phi t} u^{t}+g_{\phi \phi} u^{\phi},
\end{aligned}
$$

with ( $\tau$ being proper time)

$$
u^{\mu}=\left\{u^{t}, u^{r}, u^{\theta}, u^{\phi}\right\}=\left\{\frac{d t}{d \tau}, \frac{d r}{d \tau}, \frac{d \theta}{d \tau}, \frac{d \phi}{d \tau}\right\} .
$$

Inverting the previous relations, one obtains the angular velocity at fixed radial distance from the equator,

$$
\frac{u^{\phi}}{u^{t}}=\Omega=\frac{\ell\left[r(r-2 M)+\left(1-\beta^{2}\right) Q^{2}\right]-a e\left[\left(1-\beta^{2}\right) Q^{2}-2 M r\right]}{e r^{4}+a^{2} e\left[r(2 M+r)-\left(1-\beta^{2}\right) Q^{2}\right]+a \ell\left[\left(1-\beta^{2}\right) Q^{2}-2 M r\right]} .
$$

Time-like geodesics associated with massive particles satisfy the condition

$$
u^{\mu} u^{\nu} g_{\mu \nu}=-1
$$

To compute the radial position of stable circular time-like trajectories on the equatorial plane, we assume that $u^{\theta}=0$, and we can combine equations (44) and (47) to obtain the following expression for derivatives of the radial position of a massive particle along proper time

$$
\frac{r^{4}}{\Delta^{2}}\left[\Delta^{2}-\beta^{4} Q^{4}\right]\left(\frac{d r}{d \tau}\right)^{2}-\frac{2 \beta^{2} r^{2} Q^{2}}{\Delta}\left(a^{2} e-a \ell+e r^{2}\right)\left(\frac{d r}{d \tau}\right)-\left(a^{2} e-a \ell+e r^{2}\right)^{2}+\left(\Delta-\beta^{2} Q^{2}\right)\left(1+\frac{(a e-\ell)^{2}}{r^{2}}\right)=0 .
$$


The previous equation can be recast as

$$
\frac{\left[\Delta^{2}-\beta^{4} Q^{4}\right]}{2 \Delta^{2}}\left(\frac{d r}{d \tau}\right)^{2}-\frac{\beta^{2} Q^{2}}{r^{2} \Delta}\left(a^{2} e-a \ell+e r^{2}\right)\left(\frac{d r}{d \tau}\right)+V_{e f f(r, e, \ell)}=\frac{e^{2}-1}{2}
$$

where we have define an effective potential

$$
V_{e f f}=-\frac{M}{r}+\frac{a^{2}\left(1-e^{2}\right)+\ell^{2}+\left(1-\beta^{2}\right) Q^{2}}{2 r^{2}}-\frac{M(\ell-a e)^{2}}{r^{3}}+\frac{\left(1-\beta^{2}\right) Q^{2}(\ell-a e)^{2}}{2 r^{4}} .
$$

The effective potential above is both energy and angular momentum dependent. Equations (49), (50) are what we need to analyse corotating $(\ell>0)$ and counterrotating $(\ell<0)$ circular trajectories, associated with objects moving in the same or opposite sense of the black hole.

Before continuing, it is important to notice that the expression for the potential (50) has a structure identical to the KN case, which is recovered sending $\beta^{2} \rightarrow 0$. New opportunities arise for our case with non-linear vectortensor interactions. In the regime $\beta^{2}>1$, the relative sign among different contributions to the effective potential (50) changes with respect to standard $\mathrm{KN}$ black holes (in that case, this regime would correspond to an unphysical negative square charge). Hence this regime is interesting since it can lead to qualitatively new features for circular orbits. As a concrete example, a straightforward computation starting from eq (46) leads to the following expression for the angular velocity of massive particles on circular orbits

$$
\Omega=\frac{M}{a M \pm \frac{r^{2} M}{\sqrt{r M+Q^{2}\left(\beta^{2}-1\right)}}}
$$

with \pm indicating orbits corotating/counterrotating with the black hole. If $\beta^{2}>1$, corotating orbits spin faster than the corresponding Kerr-Newman case, for identical values of the asymptotic charges $M, Q, a$.

We now proceed examining the existence and properties of marginally stable innermost circular orbits for test particles moving on the equatorial plane of our geometry, lying on a fixed radial position $\bar{r}$ in Boyer-Lindquist coordinates. Such trajectories are called ISCO [32]. To move on a circular orbit of radius $\bar{r}$, both the initial radial velocity and the radial acceleration must vanish, hence

$$
\begin{aligned}
& V_{e f f}(\bar{r}, e, \ell)=\frac{e^{2}-1}{2}, \\
& \left.\frac{\partial V_{e f f}(r, e, \ell)}{\partial r}\right|_{r=\bar{r}}=0 .
\end{aligned}
$$

Furthermore, the condition of stability implies that the particle must be at a minimum point of the potential, namely

$$
\left.\frac{\partial^{2} V_{e f f}(r, e, \ell)}{\partial r^{2}}\right|_{r=\bar{r}} \geq 0
$$

where equality holds for the marginally stable circular trajectories that we wish to investigate. The previous three conditions lead to three equations which determine the three quantities $e, \ell, \bar{r}$ characterizing marginally stable innermost trajectories. In order to express formulae in the simplest possible way, it is convenient to rescale our quantities as follows

$$
x=\frac{\bar{r}}{M} \quad, \quad \hat{a}=\frac{a}{M} \quad, \quad q=\frac{Q^{2}}{M^{2}}\left(1-\beta^{2}\right) .
$$

Notice that $q$ can be negative, if $\beta^{2}>1$ : a negative $q$ can not be obtained in a Kerr-Newman-like configuration, and is distinctive of our disformed black hole geometry. We can re-express and solve the three equations (52)-(53) (with inequality saturated) in terms of the three unknown quantities $e, \ell, x$. After various algebraic manipulations, we get the following expressions for $e$ and $\ell$ in terms of $x$ :

$$
\begin{aligned}
e^{2} & =\frac{q(3-4 x)+x(3 x-2)}{x(3 x-4 q)}, \\
\frac{\ell^{2}}{M^{2}} & =\frac{\hat{a}^{2}(3 q-2 x)+x\left(4 q^{2}-9 q x+6 x^{2}\right)}{x(3 x-4 q)} .
\end{aligned}
$$


Only the angular momentum of the particle depends on the black hole spin $\hat{a}$ : there are two possibilities to consider, positive or negative $\ell$ - corresponding to trajectories corotating or counterrotating with respect to the black hole. The equation determining the radial position $x=\bar{r} / M$ of the ISCO is an algebraic equation of sixth degree

$$
\begin{gathered}
x^{6}-12 x^{5}-6\left(\hat{a}^{2}-3 q-6\right) x^{4}-4\left(-2 \hat{a}^{2} q+7 \hat{a}^{2}+2 q^{2}+27 q\right) x^{3}+3\left(3 \hat{a}^{4}+10 \hat{a}^{2} q+43 q^{2}\right) x^{2} \\
-24\left(\hat{a}^{4} q+4 \hat{a}^{2} q^{2}+3 q^{3}\right) x+16 q^{2}\left(\hat{a}^{2}+q\right)=0 .
\end{gathered}
$$

There are up to six real solutions to the previous equation, depending on the values of $a, q$ : fully analytical expressions are in general unavailable. We are interested to examine the case $q \leq 0$, distinctive of our black hole configuration. We demand that the ISCOs lie outside the position of the external black hole horizon, located at

$$
x \geq x_{\text {hor }}=1+\sqrt{1-\hat{a}^{2}-q} .
$$

We study corotating and counterrotating configurations for representative choices of $q$ for the rest of this section, starting from a brief review of ISCOs for Kerr black holes $(q=0)$.

- Case $q=0$ : the Kerr black hole. The case $q=0$ reduces the system to a Kerr black hole. Equation (5) simplifies considerably, and leads to two branches of physical solutions, corresponding to a corotating and a counterrotating orbit: see the textbook [32] for an excellent account. We plot in Figure 2 the radial position of the orbit $x=\bar{r} / M$ versus black hole spin $a / M$ : corotating orbits become closer and closer to the horizon as we increase the spin of the black hole (see black curve on the left panel), while counterrotating orbits become more and more distant. Corotating orbits can touch the horizon for the extremal value of the spinning parameter $a$. The binding energy of the object per unit rest mass - given by the quantity $(1-e)$ - versus black hole spin have opposite behaviour: the binding energies of corotating objects on ISCOs increase as the black hole spin increases, while the same quantity decreases for counterrotating objects. The maximal binding energy for an object in a corotating orbit on an extremal black hole, $a=M$ reaches the value $1-e=0.42$.
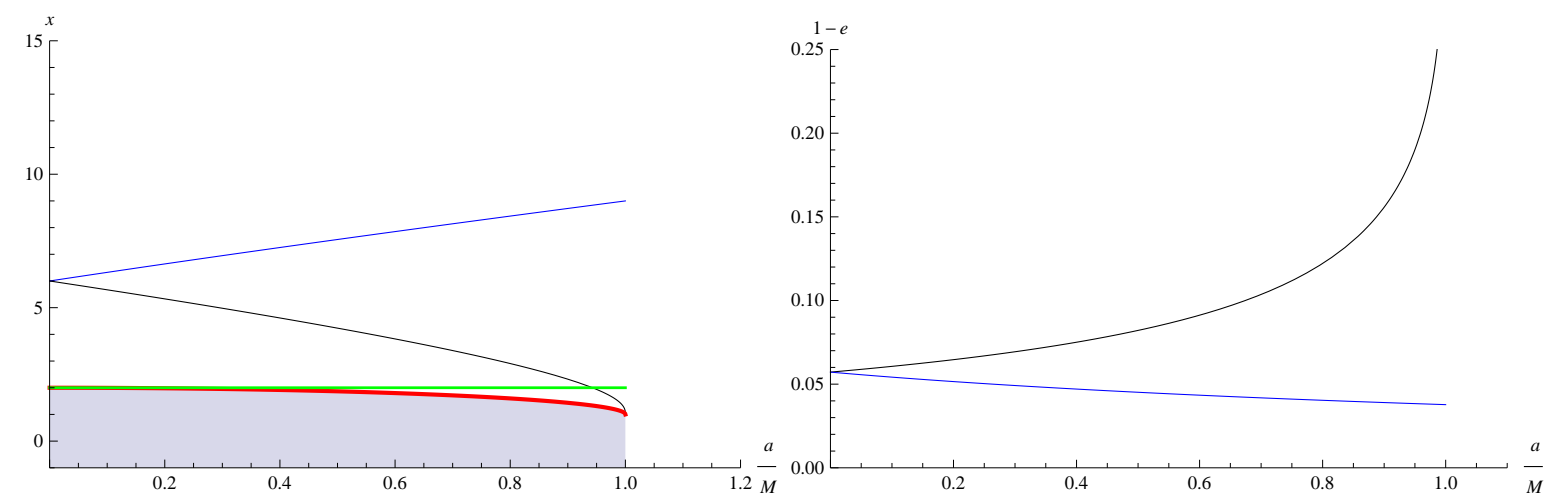

Figure 2: Panel on the left: Innermost circular orbits for a Kerr black hole: radial position of the orbit $x=\bar{r} / M$ versus black hole spin $a / M$. There are two branches of solutions, corresponding to corotating (black) and counterrotating (blue) ISCOs. The red line corresponds to the horizon, and the shaded part the forbidden region inside the black hole horizon. The green line is the boundary of the ergosphere. Panel on the right: binding energy $1-e$ versus black hole spin a/M.

- Case $q=-0.2$ : When $q$ is small and negative, the properties of ISCO trajectories are qualitatively similar to the case of Kerr. An important difference is that corotating stable orbits can neverand touch the horizon, not even for extremal values of the spinning parameter, which is $a_{\max }=1.05 M$ for $q=-0.2$. The binding energy for corotating ISCO never exceed values of order 20 per cent in this case. Notice that, as discussed in Section 4, the equatorial region of the black hole could rotate faster than $a_{\max }$, and still be well defined: we represent with dashed red curve the additional interval of black hole spin that would be allowed at the equator. On the 
other hand, the requirement of having a regular horizon everywhere, including at the poles, restricts the value of $a$ to be smaller than $a_{\max }$.
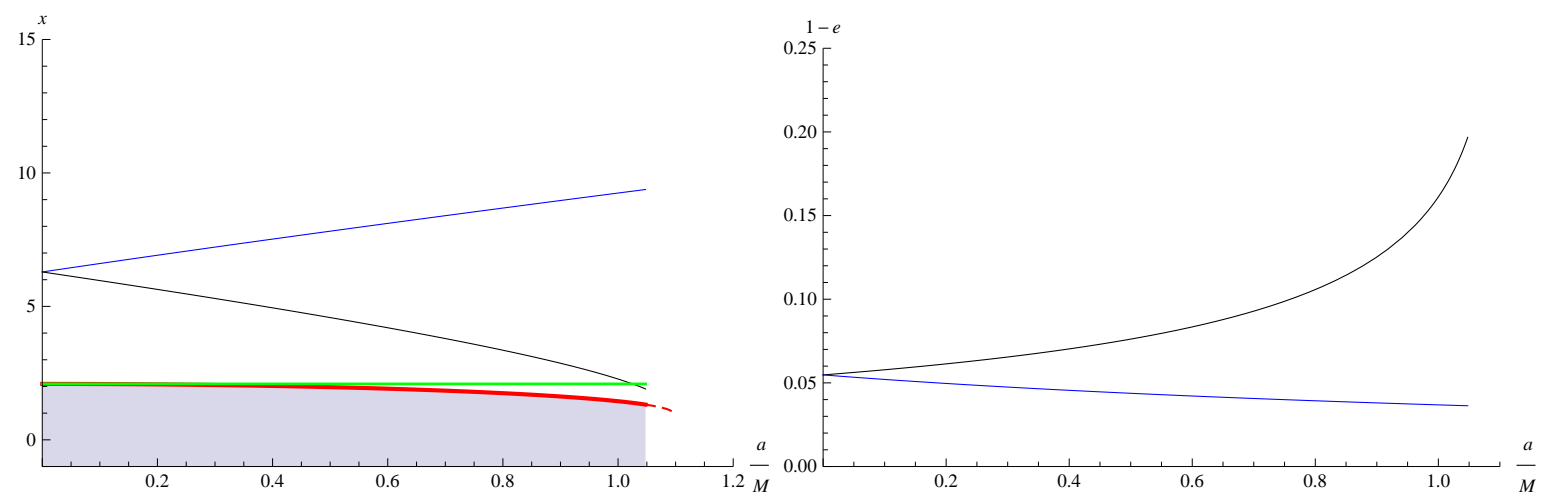

Figure 3: Innermost circular orbits (panel on the left) and binding energy versus black hole spin (panel on the right) for a black hole with $q=-0.2$. Color codes as in Figure 2. The corotating ISCO does not touch the black hole horizon, even for extremal values of the black hole spin.

- Case $q=-5$ : When $q$ becomes more negative, the features we noticed for small $q$ become more accentuated. For $q=-5$, the maximal value of the rotation parameter is $a_{\max }=1.72 M$. Corotating ISCOs are far from the black hole horizon, even for extremal values of $a_{\max }$. The maximal value of the binding energy on a corotating ISCO is of few per cent.
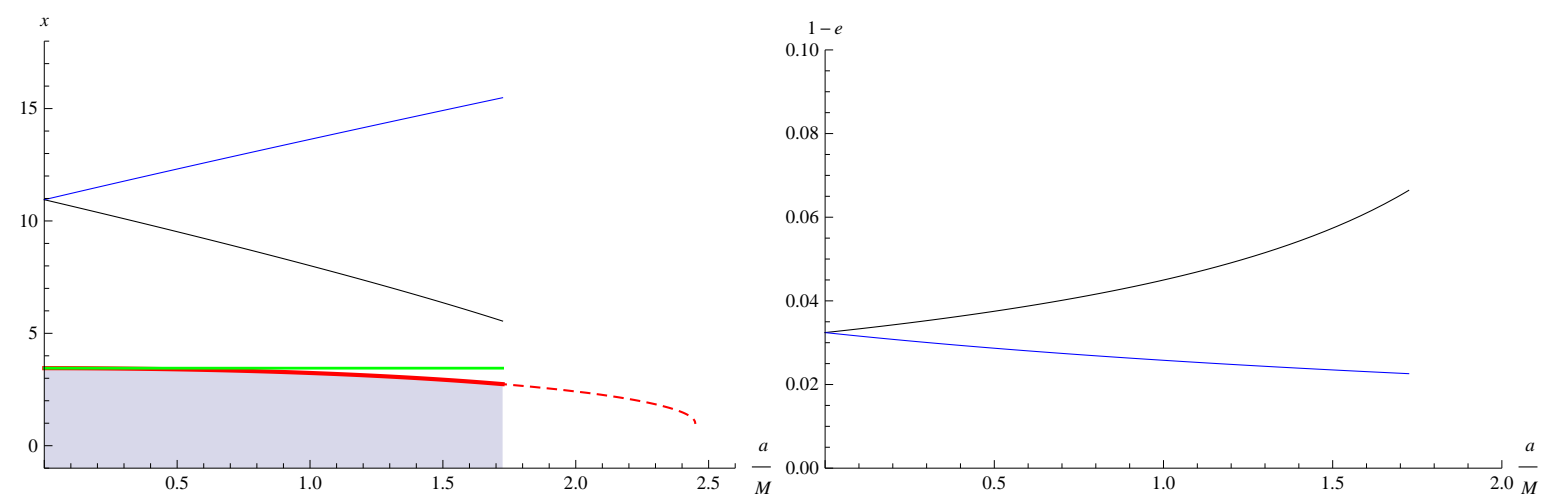

Figure 4: ISCOs (left) and binding energies (right) versus black hole spin, for a black hole with $q=-5$. Color codes as in Figure 2. 
Our findings might be relevant for studying extraction of rotational energy from the disformed black hole - a broad topic that goes outside the scope of the present paper, but that we can start to qualitatively touch here. For large values of $|\beta|$, the size of the ergoregion can be greater than for Kerr black holes: at the equator, the radial size of the ergosphere is

$$
r_{\text {erg }}=M\left(1+\sqrt{1+\frac{Q^{2}}{M^{2}}\left(\beta^{2}-1\right)}\right),
$$

hence it can be well larger than $2 M$ for $|\beta| \gg 1$. Massive particles in the ergosphere can have negative energy [32]. This implies that a Penrose process $[35,36]$ can be in principle devised: a massive object - e.g. a star - can break into two fragments through tidal effects within the ergosphere. One part - with negative energy - falls into the black hole; the other escapes, carrying away more energy than the initial object, and slowing down the black hole rotation. Since the ergosphere region can be large in our set-up, it might be easier to extract energy through this process ${ }^{1}$. Other mechanisms for energy extraction can be applied in our context, as black hole superradiance [37] or some version of the Blandford-Znajek mechanism [38], possibly using our vector interactions. In fact, it is known that the efficiency of energy extraction can be increased for charged black holes [39]. Moreover, as we have learned around equation (51), for our configurations the angular velocity of massive particles on circular orbits can be parametrically larger than in Kerr, possibly making more efficient the mechanism behind the idea of black hole colliders [40]. We plan to return to discuss these topics in a separate publication.

\section{Discussion}

In this work we determined and analysed exact solutions for rotating black holes in a specific example of vector Galileons, a theory of modified gravity motivated by the dark energy problem, and that involves additional vector degrees of freedom besides a spin-2 graviton. The set-up have many features in common with several modified gravity models, including derivative self-couplings and non-minimal couplings with gravity. We determined our new black hole solution applying an appropriate disformal transformation to a system related to the Kerr-Newman solution of Einstein-Maxwell gravity, and discussed various physical implications that differentiate this system from known rotating solutions in General Relativity.

The rotating black holes configurations we determined are valid also for large values of the black hole spin parameter. Their deviation from a Kerr-Newman solution is parametrically controlled by a dimensionless quantity associated with non-minimal couplings between vectors and gravity. The black holes are characterised by three asymptotic conserved quantities - black hole mass, spin, and vector charge. The black hole horizon is oblate in Boyer-Lindquist coordinates, since its radial position depends on the polar coordinates: this makes a difference with standard Kerr-Newman solutions, for which the horizon is at fixed radius in Boyer-Lindquist coordinates. We shown that the maximal value for the black hole spin can be parametrically larger than in the Kerr-Newman family of solutions, for the same value of asymptotic charges. We noticed that our solutions maintain a regular horizon even in the limit of zero mass for the black hole. We then studied equatorial circular trajectories around our solutions, which admit an analytical treatment. We shown that probe massive objects can rotate faster than around Kerr-Newman configurations. Innermost stable circular orbits (ISCOs) lie far away from the black hole horizon with respect to rotating black holes in GR.

Having a new family of analytic rotating solutions for a theory of modified gravity opens new opportunities for finding ways to test these theories against astrophysical or cosmological observations. It will be interesting to determine whether there exists an analogue of the Carter constant [41], which allows us to integrate the equations for time-like geodesics also beyond the case of circular equatorial orbits we studied here. The analysis of possible mechanisms for extracting rotational energy from the black hole, possibly exploiting vector interactions, deserves further study. Finally, the issue of stability of our configurations is an open issue that will need to be addressed for understanding the physical relevance of these objects.

\footnotetext{
${ }^{1}$ Although it might be not too efficient since only unstable circular orbits are contained in the ergoregion, and the falling objects might not find the correct orbital configurations to make the mechanism feasible.
} 


\section{Acknowledgments}

It is a pleasure to thank Javier Chagoya for discussions and suggestions. We are also grateful to Tsvetan Vetsov for pointing out some errors in an earlier version of this paper.

\section{A Asymptotic mass, charge and angular momentum}

For rotating configurations which admit time-like Killing vectors, as is our case, we can define conserved currents that can be used to obtain asymptotic charges: see for example [32] for details.

The gravitational energy - the mass - can be obtained starting from the time-like Killing vector $k^{\mu}$ associated with time translations, $k^{\mu}=\{1,0,0,0\}$, by defining a current as follows

$$
J^{\mu}=k_{\nu} R^{\mu \nu}
$$

Such current is conserved by means of Killing condition $\nabla_{[\mu} k_{\nu]}=0$ and the Bianchi identity. We can use it to define a conserved energy

$$
E_{B H}=\int_{\Sigma} d^{3} x \sqrt{\gamma^{(3)}} n_{\mu} J_{M}^{\mu}
$$

where $\Sigma$ is a space-like hypersurface with induced metric $\gamma_{i j}^{(3)}$ and $n_{\mu}$ is the unit normal vector to $\Sigma$. Using Stokes theorem, and the properties of Killing vectors, we can rewrite this quantity as

$$
E_{B H}=\int_{\partial \Sigma} d^{2} x \sqrt{\gamma^{(2)}} n_{\mu} \sigma_{\nu} \nabla^{\mu} k^{\nu}
$$

where the boundary $\partial \Sigma$ has metric $\gamma_{i j}^{(2)}$ and an outward-pointing normal vector $\sigma^{\mu}$. In our case, a computation of this quantity for our configuration (18) gives $E_{M}=M$, hence the parameter $M$ entering in the geometry corresponds to the gravitational mass of the object.

Analogously, we can define a conserved 'electric' charge of the object

$$
Q_{B H}=-\int_{\partial \Sigma} d^{2} x \sqrt{\gamma^{(2)}} n_{\mu} \sigma_{\nu} F^{\mu \nu}
$$

which in our case gives, as expected, $Q_{B H}=Q$, since at spatial infinity we do not expect contributions from the scalar field. In fact, the geometry does not feel the disformal transformation at spatial infinity, since the "disformal contribution" goes to infinity much faster than the GR one.

Finally, the conserved angular momentum is

$$
J_{B H}=-\frac{1}{2} \int_{\partial \Sigma} d^{2} x \sqrt{\gamma^{(2)}} n_{\mu} \sigma_{\nu} \nabla^{\mu} \varphi^{\nu}
$$

where $\varphi^{\mu}$ is a Killing vector associated with rotational symmetry around the coordinate $\phi: \varphi^{\mu}=\{0,0,0,1\}$. For our configuration we find $J_{B H}=a M$.

To sum up, we find three conserved asymptotic quantities with a clear physical interpretation, which can be easily associated with the constant parameters appearing in the geometry.

\section{References}

[1] N. Yunes, K. Yagi and F. Pretorius, Phys. Rev. D 94 (2016) no.8, 084002 doi:10.1103/PhysRevD.94.084002 [arXiv:1603.08955 [gr-qc]].

[2] T. Clifton, P. G. Ferreira, A. Padilla and C. Skordis, Phys. Rept. 513 (2012) 1 doi:10.1016/j.physrep.2012.01.001 [arXiv:1106.2476 [astro-ph.CO]].

[3] E. J. Copeland, M. Sami and S. Tsujikawa, Int. J. Mod. Phys. D 15 (2006) 1753 doi:10.1142/S021827180600942X [hep-th/0603057]. 
[4] J. Khoury and A. Weltman, Phys. Rev. Lett. 93 (2004) 171104 doi:10.1103/PhysRevLett.93.171104 [astroph/0309300].

[5] A. I. Vainshtein, Phys. Lett. 39B (1972) 393. doi:10.1016/0370-2693(72)90147-5

[6] A. Joyce, B. Jain, J. Khoury and M. Trodden, Phys. Rept. 568 (2015) 1 doi:10.1016/j.physrep.2014.12.002 [arXiv:1407.0059 [astro-ph.CO]].

[7] A. Nicolis, R. Rattazzi and E. Trincherini, Phys. Rev. D 79 (2009) 064036 doi:10.1103/PhysRevD.79.064036 [arXiv:0811.2197 [hep-th]].

[8] G. W. Horndeski, Int. J. Theor. Phys. 10 (1974) 363. doi:10.1007/BF01807638

[9] C. A. R. Herdeiro and E. Radu, Int. J. Mod. Phys. D 24 (2015) no.09, 1542014 doi:10.1142/S0218271815420146 [arXiv:1504.08209 [gr-qc]]; T. P. Sotiriou, doi:10.1088/0264-9381/32/21/214002 [arXiv:1505.00248 [gr-qc]]; M. S. Volkov, arXiv:1601.08230 [gr-qc].

[10] G. Tasinato, JHEP 1404, 067 (2014) doi:10.1007/JHEP04(2014)067 [arXiv:1402.6450 [hep-th]];

[11] B. M. Gripaios, JHEP 0410, 069 (2004) doi:10.1088/1126-6708/2004/10/069 [hep-th/0408127];

[12] L. Heisenberg, JCAP 1405, 015 (2014) doi:10.1088/1475-7516/2014/05/015 [arXiv:1402.7026 [hep-th]].

[13] J. Chagoya, G. Niz and G. Tasinato, Class. Quant. Grav. 33, no. 17, 175007 (2016) doi:10.1088/02649381/33/17/175007 [arXiv:1602.08697 [hep-th]].

[14] M. Minamitsuji, Phys. Rev. D 94 (2016) no.8, 084039 doi:10.1103/PhysRevD.94.084039 [arXiv:1607.06278 [gr-qc]]; A. Cisterna, M. Hassaine, J. Oliva and M. Rinaldi, Phys. Rev. D 94 (2016) no.10, 104039 doi:10.1103/PhysRevD.94.104039 [arXiv:1609.03430 [gr-qc]]; E. Babichev, C. Charmousis and M. Hassaine, JHEP 1705 (2017) 114 doi:10.1007/JHEP05(2017)114 [arXiv:1703.07676 [gr-qc]]; J. Chagoya, G. Niz and G. Tasinato, Class. Quant. Grav. 34 (2017) no.16, 165002 [arXiv:1703.09555 [gr-qc]]; L. Heisenberg, R. Kase, M. Minamitsuji and S. Tsujikawa, [arXiv:1705.09662 [gr-qc]]; L. Heisenberg, R. Kase, M. Minamitsuji and S. Tsujikawa, [arXiv:1706.05115 [gr-qc]]; J. Chagoya and G. Tasinato, [arXiv:1707.07951 [hep-th]]; S. Nakamura, R. Kase and S. Tsujikawa, [arXiv:1707.09194 [gr-qc]].

[15] E. Babichev and A. Fabbri, Phys. Rev. D 90 (2014) 084019 doi:10.1103/PhysRevD.90.084019 [arXiv:1406.6096 [gr-qc]].

[16] J. W. Moffat, Eur. Phys. J. C 75 (2015) no.3, 130 doi:10.1140/epjc/s10052-015-3352-6 [arXiv:1502.01677 [gr-qc]].

[17] C. A. R. Herdeiro and E. Radu, Phys. Rev. Lett. 112 (2014) 221101 doi:10.1103/PhysRevLett.112.221101 [arXiv:1403.2757 [gr-qc]].

[18] P. Pani, C. F. B. Macedo, L. C. B. Crispino and V. Cardoso, Phys. Rev. D 84 (2011) 087501 doi:10.1103/PhysRevD.84.087501 [arXiv:1109.3996 [gr-qc]].

[19] B. Kleihaus, J. Kunz, S. Mojica and E. Radu, Phys. Rev. D 93 (2016) no.4, 044047 doi:10.1103/PhysRevD.93.044047 [arXiv:1511.05513 [gr-qc]].

[20] A. Maselli, H. O. Silva, M. Minamitsuji and E. Berti, Phys. Rev. D 92 (2015) no.10, 104049 doi:10.1103/PhysRevD.92.104049 [arXiv:1508.03044 [gr-qc]].

[21] E. Berti et al., Class. Quant. Grav. 32 (2015) 243001 doi:10.1088/0264-9381/32/24/243001 [arXiv:1501.07274 [gr-qc]].

[22] T. Johannsen and D. Psaltis, Phys. Rev. D 83 (2011) 124015 doi:10.1103/PhysRevD.83.124015 [arXiv:1105.3191 [gr-qc]].

[23] R. Konoplya, L. Rezzolla and A. Zhidenko, theories of gravity," Phys. Rev. D 93, no. 6, 064015 (2016) [arXiv:1602.02378 [gr-qc]].

[24] J. D. Bekenstein, Phys. Rev. D 48 (1993) 3641 doi:10.1103/PhysRevD.48.3641 [gr-qc/9211017].

[25] D. Bettoni and S. Liberati, Phys. Rev. D 88 (2013) 084020 doi:10.1103/PhysRevD.88.084020 [arXiv:1306.6724 [gr-qc]]; M. Zumalacrregui and J. Garca-Bellido, Phys. Rev. D 89 (2014) 064046 doi:10.1103/PhysRevD.89.064046 [arXiv:1308.4685 [gr-qc]].

[26] R. Kimura, A. Naruko and D. Yoshida, JCAP 1701 (2017) no.01, 002 doi:10.1088/1475-7516/2017/01/002 [arXiv:1608.07066 [gr-qc]]. 
[27] G. Tasinato, Class. Quant. Grav. 31 (2014) 225004 doi:10.1088/0264-9381/31/22/225004 [arXiv:1404.4883 [hepth]]; A. De Felice, L. Heisenberg, R. Kase, S. Tsujikawa, Y. l. Zhang and G. B. Zhao, Phys. Rev. D 93 (2016) no.10, 104016 doi:10.1103/PhysRevD.93.104016 [arXiv:1602.00371 [gr-qc]]; C. M. Nieto and Y. Rodriguez, Mod. Phys. Lett. A 31 (2016) no.21, 1640005 doi:10.1142/S0217732316400058 [arXiv:1602.07197 [gr-qc]]; A. De Felice, L. Heisenberg, R. Kase, S. Mukohyama, S. Tsujikawa and Y. l. Zhang, JCAP 1606 (2016) no.06, 048 doi:10.1088/1475-7516/2016/06/048 [arXiv:1603.05806 [gr-qc]]; A. De Felice, L. Heisenberg, R. Kase, S. Mukohyama, S. Tsujikawa and Y. l. Zhang, Phys. Rev. D 94 (2016) no.4, 044024 doi:10.1103/PhysRevD.94.044024 [arXiv:1605.05066 [gr-qc]]; L. Heisenberg, R. Kase and S. Tsujikawa, JCAP 1611 (2016) no.11, 008 doi:10.1088/1475-7516/2016/11/008 [arXiv:1607.03175 [gr-qc]]; S. Nakamura, R. Kase and S. Tsujikawa, Phys. Rev. D 95 (2017) no.10, 104001 doi:10.1103/PhysRevD.95.104001 [arXiv:1702.08610 [gr-qc]]. A. de Felice, L. Heisenberg and S. Tsujikawa, Phys. Rev. D 95 (2017) no.12, 123540 doi:10.1103/PhysRevD.95.123540 [arXiv:1703.09573 [astro-ph.CO]].

[28] N. Khosravi, Phys. Rev. D 89 (2014) no.12, 124027 doi:10.1103/PhysRevD.89.124027 [arXiv:1404.7503 [hep-th]]; P. Fleury, J. P. Beltran Almeida, C. Pitrou and J. P. Uzan, JCAP 1411 (2014) no.11, 043 doi:10.1088/14757516/2014/11/043 [arXiv:1406.6254 [hep-th]]; M. Hull, K. Koyama and G. Tasinato, JHEP 1503 (2015) 154 doi:10.1007/JHEP03(2015)154 [arXiv:1408.6871 [hep-th]]; M. Hull, K. Koyama and G. Tasinato, Phys. Rev. D 93 (2016) no.6, 064012 doi:10.1103/PhysRevD.93.064012 [arXiv:1510.07029 [hep-th]]; F. Charmchi, Z. Haghani, S. Shahidi and L. Shahkarami, Phys. Rev. D 93 (2016) no.12, 124044 doi:10.1103/PhysRevD.93.124044 [arXiv:1511.07034 [hep-th]]; J. Chagoya and G. Tasinato, JHEP 1602 (2016) 063 doi:10.1007/JHEP02(2016)063 [arXiv:1511.07805 [hep-th]]; L. Heisenberg, R. Kase and S. Tsujikawa, Phys. Lett. B 760 (2016) 617 doi:10.1016/j.physletb.2016.07.052 [arXiv:1605.05565 [hep-th]]; A. Amado, Z. Haghani, A. Mohammadi and S. Shahidi, Phys. Lett. B 772 (2017) 141 doi:10.1016/j.physletb.2017.06.040 [arXiv:1612.06938 [hep-th]]; J. Bonifacio, K. Hinterbichler and R. A. Rosen, Phys. Rev. D 94 (2016) no.10, 104001 doi:10.1103/PhysRevD.94.104001 [arXiv:1607.06084 [hep-th]]; E. Allys, P. Peter and Y. Rodriguez, JCAP 1602 (2016) no.02, 004 doi:10.1088/14757516/2016/02/004 [arXiv:1511.03101 [hep-th]]; J. Beltran Jimenez and L. Heisenberg, Phys. Lett. B 757 (2016) 405 doi:10.1016/j.physletb.2016.04.017 [arXiv:1602.03410 [hep-th]].

[29] S. A. Teukolsky, Class. Quant. Grav. 32 (2015) no.12, 124006 doi:10.1088/0264-9381/32/12/124006 [arXiv:1410.2130 [gr-qc]].

[30] T. Adamo and E. T. Newman, Scholarpedia 9 (2014) 31791 doi:10.4249/scholarpedia.31791 [arXiv:1410.6626 [gr-qc]].

[31] J. M. Martín-Garcia, http://www.xact.es.

[32] J. B. Hartle, "An introduction to Einstein's general relativity," San Francisco, USA: Addison-Wesley (2003)

[33] R. C. Myers and M. J. Perry, Annals Phys. 172 (1986) 304. doi:10.1016/0003-4916(86)90186-7

[34] J. M. Bardeen, W. H. Press and S. A. Teukolsky, Astrophys. J. 178 (1972) 347. doi:10.1086/151796

[35] R. Penrose Riv. Nuovo Cim. , Numero Speciale 1252 ?276 (1969)

[36] R. Penrose R and R. M. Floyd R M Nature 229 177?179 (1971)

[37] Y. Zeldovich, Sov. Phys. JETP Lett. 14180 (1971)

[38] R. D. Blandford and R. L. Znajek, Mon. Not. Roy. Astron. Soc. 179 (1977) 433.

[39] M. Bhat, S. Dhurandhar, and N. Dadhich, J. Astrophys. Astron. 6, 85 (1985).

[40] M. Banados, J. Silk and S. M. West, Phys. Rev. Lett. 103 (2009) 111102 doi:10.1103/PhysRevLett.103.111102 [arXiv:0909.0169 [hep-ph]].

[41] B. Carter, Phys. Rev. 174 (1968) 1559. doi:10.1103/PhysRev.174.1559 